\title{
TINTIN22, SARKOO, DOMI ET D'AUTRES... À PROPOS DES ANTHROPONYMES FORMANT LES PSEUDONYMES
}

\author{
Jan Lazar
}

\begin{abstract}
This article focuses on anthroponyms forming the nicknames used by Internet users, particularly in real time communication on the Internet, i.e. chat communication. The main objective is to suggest a classification of nicknames based on an anthroponym and bring their exact quantification in this particular type of electronic communication. The richness of nicknames is illustrated by concrete examples taken from the corpus.

Keywords: Internet; chat; nickname; anthroponym.

Résumé: Cet article s'intéresse aux anthroponymes qui forment les pseudonymes utilisés par les internautes, notamment dans le cadre des échanges en situation de temps réel sur Internet, c'est-à-dire sur les tchats. Le présent article voudrait proposer une classification des pseudonymes créés à partir d'un anthroponyme ainsi qu'apporter leur quantification précise dans ce type de communication électronique. L'énorme richesse des pseudonymes est illustrée par des exemples concrets tirés de notre corpus.
\end{abstract}

Mots-clés : Internet ; chat ; pseudonyme ; anthroponyme.

\section{Introduction}

Le tchat, outil de communication synchrone le plus connu, est un moyen grâce auquel les internautes peuvent communiquer en temps quasi réel par l'écrit. Pour pouvoir participer à ce type de communication, les internautes doivent s'enregistrer et, en même temps, se créer une nouvelle identité virtuelle qui est représentée par des pseudonymes (nickname). Dès que le tchateur est enregistré, il peut accéder aux différents salons de discussion et participer librement, en privé ou en public, à la communication synchrone. La personnalité individuelle ${ }^{1}$ de chaque tchateur est représentée par les pseudonymes qui apparaissent dans la marge gauche de chaque salon. Vu leur énorme diversité, il nous paraît nécessaire d'en proposer un classement. Étant donné que notre précédente étude s'intéressait notamment aux pseudonymes toponymiques (Lazar : à paraître), nous voudrions orienter notre attention avant tout vers les anthroponymes formant ces pseudonymes. L'intention de notre modeste étude est de proposer une classification

\footnotetext{
$\overline{1}$ Il est à noter qu'un pseudonyme ne peut être utilisé que par un seul tchateur.
} 
et une quantification des pseudonymes anthroponymiques rencontrés dans ce type de communication.

Puisque cet environnement communicatif représente un milieu amical et spontané où on peut parler vers l'autre, quel que soit son âge, son sexe ou son milieu social (Dejond Mercier 2002 : 30), nous nous attendons à un pourcentage élevé d'anthroponymes représentés par des formes hypocoristiques, qui expriment une intention affectueuse, et ainsi soulignent le caractère convivial de cet environnement. Au contraire, nous supposons que les anthroponymes représentés par des noms complets, c'est-à-dire nom et prénom, seront assez réduits, car l'anonymat absolu reste une des caractéristiques principales de cet environnement communicatif (Jandová - David 2006 : 45). Pour réfuter ou confirmer nos hypothèses, nous allons nous servir de corpus présenté dans le paragraphe suivant.

\section{Présentation du corpus}

Le choix d'un corpus équivalent représentait un pas d'une importance cruciale pour notre recherche. Étant donné que le réseau Internet dispose d'un grand nombre de tchats, nous avons défini plusieurs critères pour notre choix :

1. Le tchat doit être destiné à un public francophone et ses discussions doivent se dérouler en français. Ceci est une condition essentielle pour tout linguiste analysant la langue française telle qu'elle est employée par les locuteurs natifs. Nos tchats sont tous placés sur un serveur français et ils ne sont pratiquement connus que dans le monde francophone. Nous en déduisons que la quasi-totalité des tchateurs est francophone et que le risque qu'un tchateur non francophone s'y glisse est largement éliminé.

2. Le tchat doit disposer d'un volume appréciable d'utilisateurs connectés (1071 pseudonymes).

3. Le chat doit être gratuit et public. Le tchat doit disposer de plusieurs salons de conversation. Étant donné que nous voulons notamment observer les discussions des jeunes internautes, le tchat doit disposer d'un salon de conversation pour les jeunes (Diskut - salle " moins de 18 ans », Adoskuat - salle " Tchat entre ados », Chat - salle « Ados »).

4. Il s'agit d'un tchat général et non modéré. Les discussions ne s'orientent pas sur un thème particulier et elles ne sont pas dirigées par un modérateur qui distribue les tours de la parole.

5. Les conversations des tchats sont librement disponibles à l'enregistrement.

D'après les critères mentionnés, nous avons choisi trois tchats qui constituent notre corpus, il s'agit des tchats : Diskut, ${ }^{2}$ Adoskuat, ${ }^{3}$ Chat. ${ }^{4}$

Nous nous sommes connecté à chacun de ces trois tchats deux fois, à des dates et heures différentes, pour pouvoir capturer le matériel le plus équilibré possible. Pour entrer dans les salons destinés aux jeunes tchateurs, nous nous sommes créé l'identité fictive d'un jeune Parisien qui utilisait le surnom Olomouc. Même si plusieurs tchateurs nous ont adressé la parole, nous n'avons engagé la conversation avec aucun d'entre eux et nous n'avons envoyé de messages ni publics ni privés. Notre objectif était d'observer et d'enregistrer des conversations spontanées, sans que les tchateurs soient informés de

\footnotetext{
2 http://www.discut.fr.

http://www.adoskuat.com/zone-chat.html.

4 http://www.chat-fr.org.
} 
notre recherche. Pendant nos six sessions, nous avons rassemblé au total 1071 pseudonymes à étudier.

\section{Pseudonymes anthroponymiques}

Les paragraphes qui suivent s'intéressent aux pseudonymes qui sont créés à partir d'un anthroponyme. Le mot provient du grec ancien et se compose d'anthrôpos qui signifie « homme » et onoma qui signifie « nom ». Rappelons qu'anthroponyme est un mot qui désigne un nom de personne, de manière générale. Il peut aussi bien désigner un prénom qu'un nom de famille, surnom, etc. (Lotko 2003 : 16). Nous nous rendons compte que le terme pseudonyme anthroponymique est déjà un pléonasme terminologique, car les pseudonymes eux-mêmes sont des anthroponymes, pourtant, pour des raisons pratiques, nous préférons utiliser ce terme pour le mettre en contraste avec les pseudonymes toponymiques que nous avons observés dans nos études précédentes.

Dans notre corpus, composé de 1071 pseudonymes, nous avons relevé 240 pseudonymes créés à partir d'un anthroponymes, ce qui représente $22,4 \%$ de tous les pseudonymes observés. Vu leur énorme diversité, nous avons décidé d'élaborer leur typologie avec une quantification précise.

\subsection{Typologie des pseudonymes anthroponymiques}

En reprenant le classement de David (Jandová - David 2006 : 71), nous avons décidé de classer les pseudonymes anthroponymiques formant notre corpus en 4 catégories.

a) La première catégorie est représentée par les pseudonymes créés à partir de prénoms dans leur forme standard (Zoe, cédric, eric, davide, etc.). Dans notre corpus nous avons trouvé 72 exemples, ce qui représente $30 \%$.

Il arrive qu'un prénom dans sa forme standard puisse déjà être pris par un autre tchateur. Si tel est le cas, nous notons que d'autres signes graphiques ou lettres s'y ajoutent pour le modifier légèrement.

p.a.s.c.a.l.e., $N-a-d-i-n-e$, Robert_, mélaniexxx, célineeee, danielA

Puisqu'une des caractéristiques principales de la communication médiée par ordinateur est l'économie de l'expression (Chovancová 2008 : 96), il se peut que les prénoms standard soient affectés par des néographies phonétisantes qui font apparaître une nouvelle graphie pour un signifiant déjà existant (Lazar 2012 : 21). Parmi ces procédés de réduction, nous notons la réduction de graphème $\mathrm{qu} \rightarrow[\mathrm{k}]$ en $\mathrm{k}$, éventuellement $\mathrm{c}[\mathrm{k}] \rightarrow \mathrm{k}$.

\section{Enrique $\rightarrow$ enrik}

Nicolas [nikola] $\rightarrow$ nikola (ce pseudonyme est aussi affecté par la graphie simplificatrice qui supprime les lettres non prononcées)

La dernière néographie qui influence les prénoms standard est le syllabogramme, qui profite de la valeur phonétique de certaines lettres de l'alphabet.

Enrique $\rightarrow$ Nrik, Thérèse $\rightarrow$ Trese

b) La deuxième catégorie est représentée par les prénoms en forme hypocoristique. Par forme hypocoristique, nous entendons une forme qui exprime une intention affectueuse (Lotko 2003 : 46 ). Étant donné que la communication sur les tchats a un caractère amical et spontané (David - Jandová 2006 : 74), les tchateurs choisissent souvent des pseudonymes en forme hypocoristique pour souligner ainsi la convivialité dans les salons 
de discussions. Les anthroponymes hypocoristiques représentent $50 \%$ de notre corpus, c'est-à-dire 120 exemples. Nous notons que les formes hypocoristiques sont généralement obtenues par une simple troncation et, dans la grande majorité, elles sont dissyllabiques.

Dominique $\rightarrow$ Domi, Judith $\rightarrow$ Judi

Néanmoins, il faut préciser que dans notre corpus nous observons aussi des formes hypocoristiques monosyllabiques. Il s'agit notamment des noms qui se terminent par une consonne.

$$
\text { Jennifer } \rightarrow \text { Jenn, Mélanie } \rightarrow \text { Mél }
$$

Il est important d'ajouter que ces hypocoristiques à caractère diminutif peuvent être redoublés. Précisons que la grande majorité des hypocoristiques à redoublement emprunte au prénom d'origine sa première syllabe (Plainat - Huerta 2006 : 341).

Dominique $\rightarrow$ Dodo, Thomas $\rightarrow$ Toto

Il est à noter que dans les hypocoristiques redoublés, les voyelles préférées sont les voyelles fermées et/ou arrondies, qui remplissent la fonction de première attaque (Plainat - Huerta 2006 : 342). La consonne [r] est incapable de fournir cette bonne attaque, ce qui explique que Robert donne Bébert plus souvent que, par ex., Roro (Plainat - Huerta 2006 : 341). Dans ce cas, ce sont le plus souvent deux éléments contigus qui sont retenus pour former un hypocoristique.

Robert $\rightarrow$ Bébert

Ajoutons que la voyelle [a], qui une valeur péjorative, se redouble rarement dans les hypocoristiques (Plainat - Huerta 2006 : 342). Ce qui explique que Marie ne donne pas Mama, mais on a relevé les pseudonymes Mimi45 ou Mémé.

c) La troisième catégorie est formée par les noms complets. Cette catégorie représente seulement $4 \%$ de notre corpus (10 exemples) et confirme ainsi notre hypothèse sur l'anonymat absolu qui est préféré par les tchateurs (Jandová - David 2006 : 45). Étant donné qu'on ne peut pas connaître la vraie identité des tchateurs, il est possible que ces noms complets soient aussi fictifs.

Julie_laval, Robert.Merle, JacquesLefebre

d) La dernière catégorie est représentée par les anthroponymes de personnages célèbres et les pseudoanthroponymes. Cette catégorie représente $16 \%$ de notre corpus, c' est-à-dire 38 exemples. L'anthroponyme le plus répandu dans cette catégorie est sans doute le nom de l'ancien président de la République française - Nicolas Sarkozy. Nous avons trouvé dans notre corpus 7 pseudonymes contenant son nom : sarky, sarko, sarkoo, sarkozy, sarka, sarkoozy, sakozy, ce qui manifeste l'énorme popularité de ce nom parmi les jeunes tchateurs. Pourtant, il faut préciser que le suffixe $-a($ sarkozy $\rightarrow$ sarka $)$ ajoute à ce pseudonyme une connotation péjorative.

En ce qui concerne les pseudoanthroponymes, nous notons la préférence des jeunes tchateurs pour les héros et héroïnes de bandes dessinées. Ainsi, nous relevons dans notre corpus : robert Bidochon, Tintin22, LAstroumphette, milouuu qui proviennent de bandes dessinés connues dans le monde francophone.

\section{Conclusion}

L'objectif de notre étude était de décrire en détail les pseudonymes qui sont créés à partir d'un anthroponyme. Pour résumer, nous avons montré que ce sont notamment 
Tintin22, Sarkoo, Domi et d'autres... À propos des anthroponymes formant les pseudonymes

les formes hypocoristiques qui sont les plus répandues dans notre corpus. En effet, il confirme notre hypothèse sur le caractère convivial de cet environnement communicatif. Les formes hypocoristiques ajoutent aux anthroponymes une connotation affectueuse et accentuent ainsi l'aspect amical et spontané de ce type de communication. Il faut préciser que les hypocoristiques peuvent être redoublés, ce qui rapproche cette communication de la langue parlée informelle.

Nous pouvons constater que l'anonymat, qui reste caractéristique pour ce type de communication, influence considérablement le choix des pseudonymes. Les noms complets, y compris fictifs, dévoilent trop la personnalité des tchateurs et, pour cette raison, ils se limitent dans notre corpus à seulement 10 exemples.

En outre, il faut ajouter que les pseudonymes anthroponymiques respectent aussi le caractère abréviatif des discussions tchatées. On note dans notre corpus des pseudonymes qui sont affectés par des néographies phonétisantes, qui réduisent le nombre de graphème (nicolas $\rightarrow$ niko, enric $\rightarrow$ Nrik). Ces pseudonymes sont souvent préférés par les tchateurs car ils abrègent le mot et, en même temps, attirent l'attention des autres participants par leur originalité.

\section{Bibliographie}

Chovancová, Katarína (2008), Les discussions en direct sur Internet (Énonciation et graphie), Banská Bystrica : Univerzita Mateja Bela.

Dejond, Aurélia - Mercier, Jacques (2002), La cyberl@ngue française, Bruxelles : La Renaissance du Livre.

JANDOvÁ, Eva - DAvid, Jaroslav (2006), Čeština na WWW chatu, Ostrava : Ostravská univerzita v Ostravě.

JANDOVÁ, Eva (2006), Konverzace na WWW chatu, Ostrava: Ostravská univerzita v Ostravě.

LAZAR, Jan (2012), «Quelques observations sur les néographies phonétisantes en français Tchaté », Linguistica pragensia XXII, 18-28.

LAZAR, Jan (à paraître), "Vers une classification toponymique des pseudonymes en français tchaté », Mikrotoponimia i makrotopominia : UL.

Lотко, Edvard (2003), Slovník ligvistických termínů pro filology, Olomouc : Univerzita Palackého v Olomouci.

PANCKHURST, Rachel (2006), Le discours électronique médié : bilan et perspectives, in: Piolat, Annie (éd.), Lire, écrire, communiquer et apprendre avec Internet, Marseille : Éditions Solal, 345-366.

PIEROZAK, Isabelle (2003), " La variation à la marge sur Internet: pseudo et (re) présentations », Cahiers du français contemporain 8, 195-222.

Plenat, Marc - Huerta, Pancho Solares (2006), « Domi, Seb, Flo et toute la famille », Cahiers de Grammaire 30, 339-357.

Jan Lazar

Katedra romanistiky

Filozofická fakulta

Ostravská univerzita

Tř. Československých legií 9

70103 Ostrava

République tchèque

Jan.lazar@osu.cz 